\title{
Comparative study of maternal and foetal outcome in instrumental vaginal deliveries
}

\author{
Mariyam S. Ahmed*, Anand N. Bhalerao
}

Department of Obstetrics and Gynecology, B. J. Government Medical College, Pune, Maharashtra, India

Received: 18 August 2017

Revised: 18 September 2017

Accepted: 25 September 2017

\section{*Correspondence:}

Dr. Mariyam S. Ahmed,

E-mail: mariyambj375@gmail.com

Copyright: () the author(s), publisher and licensee Medip Academy. This is an open-access article distributed under the terms of the Creative Commons Attribution Non-Commercial License, which permits unrestricted non-commercial use, distribution, and reproduction in any medium, provided the original work is properly cited.

\begin{abstract}
Background: The art of instrumental vaginal delivery is fading as more and more obstetricians resort to caesarean sections. Instrumental deliveries are deliveries accomplished with the use of vacuum device or forceps. Once either is applied to the foetal head, outward traction generates forces that augment maternal pushing to deliver the foetus vaginally. Objective of present study was to compare the maternal and foetal outcome of forceps versus ventouse delivery.

Methods: It was a prospective observational study conducted in Sassoon Hospital between January 2014 to January 2015. A total of 120 cases, 60 each of forceps and ventouse, admitted in labour room were included in the study. Maternal and neonatal morbidity were compared in terms of perineal lacerations, episiotomy extension, post-partum haemorrhage, Apgar score, instrumental injuries. Chi square test and z test of proportion was used to analyze the data. Results: Maternal morbidity, namely episiotomy extension as well as perineal tears were significant in the forceps group. With regards to neonatal morbidity, no statistically significant difference was noted.

Conclusions: Vacuum and forceps should remain appropriate tools in the armamentarium of the modern obstetrician. However, ventouse may be chosen first (if there is no foetal distress) as it is significantly less likely to injure the mother.
\end{abstract}

Keywords: Forceps, Outcome, Perineal tear, Ventouse

\section{INTRODUCTION}

The art of instrumental vaginal delivery is fading as more and more obstetricians resort to caesarean sections whenever they encounter any problem in the second stage, with overriding concerns for the neonate.

At times, it may be a good decision, and a safer decision, but not always. Increased second stage caesarean sections have a definite increased maternal morbidity and do not always give the neonate any appreciable advantage. ${ }^{1}$ The first instrumental deliveries were performed to extract foetuses from women at high risk of dying due to prolonged or obstructed labour. In these cases, saving the mother's life was the main priority.

With the development of safer techniques for vaginal extraction, however, these procedures now focus on safeguarding the wellbeing of the fetus. ${ }^{2}$

Operative vaginal deliveries are deliveries accomplished with the use of vacuum device or forceps. Once either is applied to the foetal head, outward traction generates forces that augment maternal pushing to deliver the foetus vaginally. The most important function of both devices is traction. ${ }^{3}$ 
However, improper and inexperienced usage of these instruments can lead to disastrous results and maternal and foetal complications. Forceps, vaccuum, and caesarean sections are relatively recent additions to the obstetrician's armamentarium. ${ }^{4}$

Objectives of present study were to find out the indications of instrumental vaginal delivery, to compare the maternal outcome of forceps and ventouse delivery and compare the foetal outcome of forceps and ventouse delivery.

\section{METHODS}

A total of 120 cases (60 each of forceps and ventouse) were included in this prospective study carried out from Jan 2014 to Jan 2015. Women attending the labour room with singleton pregnancy and vertex presentation and who were delivered by forceps or ventouse were included in this study.

After case selection, written and informed consent was taken, prerequisites fulfilled and women were randomly assigned for either vacuum or forceps application. Regardless of the ultimate mode of delivery, for the purpose of analysis, the women remained in the group to which they were originally allocated. The trial protocol was approved by the ethics committee of the institute. These patients were studied and evaluated in details with proper history taking, clinical examination and relevant investigations. Silastic cup was used in vacuum extraction. Forceps deliveries were performed using short curved outlet Wrigley's forceps. Maternal morbidity was studied in terms of cervical tears, vaginal lacerations, episiotomy extension, perineal tears, PPH, retention of urine, etc. Neonatal morbidity was studied in terms of Apgar score, instrumental injuries, cephalhematoma, NICU admission and the outcome was compared.
Chi square test and $\mathrm{z}$ test of proportion was used to analyze the data.

\section{Inclusion criteria}

- Primigravida women in labour with vertex presentation who will be delivered by forceps or vacuum application

- Multigravida with previous caesarean section or any other indication for forceps/ventouse delivery

- Preterms who will be delivered vaginally by forceps.

- Non reassuring fetal heart rate pattern in $2^{\text {nd }}$ stage of labour

- All prerequisites of instrumental vaginal deliveries being fulfilled.

\section{Exclusion criteria}

- Cephalopelvic disproportion

- Contracted pelvis

- Multifetal pregnancy

\section{RESULTS}

In the present study, foetal distress was the most common indication for application of forceps. Foetal distress was seen in $33.34 \%$ cases.

The next commonest indication is prophylactic (33.33\%) which includes previous LSCS-13.33\%, preeclampsia$5 \%$, prematurity-5\%, anaemia-5\% and heart disease-5\%.

The next most common indication was prolonged second stage of labour (i.e., where delivery is delayed for more than 2 hours in primigravida and 1 hour in multigravida after full dilatation of the cervix), in $20 \%$ cases followed by maternal exhaustion which was seen in $13.33 \%$.

Table 1: Indications of instrumental delivery.

\begin{tabular}{|lllll|l|}
\hline Indication & No. of forceps & No. of ventouse & $\%$ of forceps & $\%$ of ventouse & P value \\
\hline Prolonged second stage & 12 & 10 & 20 & 16.67 & 0.8135 \\
\hline Foetal distress & 20 & 14 & 33.34 & 23.33 & 0.3111 \\
\hline Maternal exhaustion & 8 & 10 & 13.33 & 16.67 & 0.7982 \\
\hline Previous LSCS & 8 & 10 & 13.33 & 16.67 & 0.7982 \\
\hline Preeclampsia & 3 & 10 & 5 & 16.67 & 0.0780 \\
\hline Anaemia & 3 & 5 & 5 & 8.33 & 0.5808 \\
\hline Heart disease & 3 & 1 & 5 & 1.66 & \\
\hline Preterm & 3 & 0 & 5 & 0 & 100 \\
\hline Total & 60 & 60 & 100 & & \\
\hline
\end{tabular}

$\mathrm{P}$ value $>0.05$, hence no statistical difference in the indications for ventouse and forceps.

In current study, the most common indication for ventouse application was prophylactic to cut short the second stage of labour (43.33) which includes $16.67 \%$ previous LSCS, $16.67 \%$ preeclampsia, $8.33 \%$ anaemia and $1.66 \%$ heart disease), followed by foetal distress which was seen in $23.33 \%$ cases. 
Table 1 compares the indications of forceps versus ventouse delivery. As seen, forceps was the preferred instrument in cases of foetal distress and preterm, whereas ventouse was preferred in prophylactic cases to cut short short the second stage of labour.

Table 2: Maternal complications.

\begin{tabular}{|c|c|c|c|c|c|}
\hline Complication & No. of forceps & No. of ventouse & $\%$ of forceps & $\%$ of ventouse & $P$ value \\
\hline Extension of episiotomy & 3 & 1 & 5 & 1.67 & \\
\hline Vaginal laceration & 3 & 2 & 5 & 3.33 & \\
\hline Cervical tear & 1 & 0 & 1.67 & 0 & \\
\hline Perineal tear ( $3^{\text {rd }}$ degree $)$ & 2 & 0 & 3.33 & 0 & \\
\hline Traumatic PPH & 1 & 0 & 1.67 & 0 & \\
\hline Total trauma & 10 & 3 & 16.67 & 5 & 0.019 \\
\hline
\end{tabular}

$\mathrm{P}$ value obtained by $\mathrm{z}$ test of proportion $<0.05$, which shows that there is a statistically significant difference in the maternal complications with forceps compared to ventouse.

Table 2 shows that extension of episiotomy was observed in 3 cases $(5 \%)$ of forceps delivery. The incidence of vaginal lacerations was $5 \%$. These lacerations occur if the blades slip off or if undue force is used while inserting the blades. Cervical tear was reported in $1.67 \%$ cases. Each cervix was explored after application of forceps. These tears were sutured in the operation theatre. 1 case of cervical tear resulted in traumatic postpartum haemorrhage. There were no cases of atonic PPH. Third degree perineal tear was reported in 2 cases. There were no cases of complete perineal tear. These tears were sutured immediately and postoperative period was uneventful. Thus, proper perineal support, slow extension of the head, and allowing the increasing diameters of the foetal head to pass through the vulva gradually, prevents perineal injuries.

In case of ventouse delivery, extension of episiotomy was reported in 1 case (1.67\% incidence), vaginal lacerations were seen in $3.33 \%$ of the cases. There were no cases of cervical tear, perineal tear, atonic or traumatic postpartum haemorrhage in the present study. Table 2 shows that maternal complications like extension of episiotomy, vaginal lacerations, cervical tear and perineal tears were more common with forceps delivery compared to ventoue delivery.

Table 3: Puerperal complications.

\begin{tabular}{|llllll|} 
Complication & No. of forceps & No. of ventouse & $\%$ of forceps & \% of ventouse & P value \\
\hline Retention of urine & 1 & 0 & 1.67 & 0 & 0.317 \\
\hline Infection of episiotomy & 1 & 1 & 1.67 & 1.67 & \\
\hline UTI & 1 & 0 & 1.66 & 0 & 0.317 \\
\hline Nil & 57 & 59 & 95 & 98.33 & \\
\hline
\end{tabular}

$\mathrm{P}$ value $>0.05$, hence no significant difference in the puerperal complications between forceps and ventouse

Table 3 shows the puerperal complications with instrumental vaginal delivery. In both the groups, infection of episiotomy wound was seen in 1 case each $(1.67 \%)$ Both the cases resulted in episiotomy wound gape and resuturing was required. It was probably due to improper hygiene maintained by the patients.

1 case of urinary retention was seen in the forceps group. Urine retention occurs due to the pain and reflex inhibition of urine sensation.

Table 3 shows the puerperal complications. Infection of episiotomy occurred in both the groups, whereas retention of urine was observed in 1 case in the forceps group.

Table 4 demonstrates the foetal complications associated with instrumental delivery. In the forceps group, scalp injury was seen in 1 case $(1.67 \%)$, while superficial bruises and lacerations were seen in 3 cases $(5 \%)$. These were treated by keeping them dry and treating the baby with systemic antibiotics. There were no cases of cephalhematoma or neonatal jaundice. 1 case of neonatal mortality was reported. The mortality was due to respiratory distress and cannot be directly attributed to forceps application. 
Table 4: Foetal complications.

\begin{tabular}{|llllll|}
\hline Complication & No of forceps & No of ventouse & $\%$ of forceps & $\%$ of ventouse & P value \\
\hline Scalp injury & 1 & 0 & 1.67 & 0 & 0.31 \\
\hline Abrasions and bruises & 3 & 1 & 5 & 1.67 & 0.302 \\
\hline Cephalhematoma & 0 & 2 & 0 & 3.33 & 0.152 \\
\hline Neonatal jaundice & 0 & 1 & 0 & 1.67 & 0.31 \\
\hline Neonatal death & 1 & 0 & 1.67 & 0 & 0.31 \\
\hline Nil & 55 & 56 & 91.66 & 93.33 & 100 \\
\hline Total & 60 & 60 & 100 & & \\
\hline
\end{tabular}

$P$ value $>0.05$, hence not significant

In the ventouse group, superficial bruises and lacerations were reported in $1.67 \%$ cases. Incidence of cephalhematoma in the present study was $3.33 \%$ and that of neonatal jaundice was $1.67 \%$. Neonatal jaundice was treated by phototherapy. No cases of convulsions or neonatal death. Also, there were no complications like intraventricular, subgaleal or subconjunctival haemorrhage. Table 4 shows that foetal complications like scalp injury and abrasions and bruises are more prevalent in the forceps group, while cephalhematoma and neonatal jaundice was more common in the ventouse group.

Table 5: APGAR score.

\begin{tabular}{|lllll|l|}
\hline APGAR score & No. of forceps & No. of ventouse & $\%$ of forceps & \% of ventouse & P value \\
\hline $7--10$ & 47 & 53 & 78.33 & 88.33 & 0.2207 \\
\hline $4--6$ & 13 & 7 & 21.67 & 11.67 & 0.2207 \\
\hline Total & 60 & 60 & 100 & 100 & \\
\hline
\end{tabular}

$P$ value $>0.05$, hence not significant.

Table 5 shows the APGAR values of the babies delivered by instrumental delivery. $78.33 \%$ of cases delivered by forceps and $88.33 \%$ of cases delivered by ventouse had APGAR score between 7-10.

\section{DISCUSSION}

In the present study, the most common indication for forceps was fetal distress seen in $33.34 \%$ of the cases (as seen in Table 1 and Figure 1), whereas in ventouse it was prophylactic to cut short second stage of labour, seen in $26 \%$ cases as seen in Table 1 . However, there is no statistical difference in the indications between the two as seen in Table 1. These results are similar to that obtained by Abha and Vaishnav. ${ }^{5,6}$

The maternal trauma was comparatively less in the ventouse group compared to forceps group as seen in Table 2 and Figure 2. Similar results have been reported by Abha's study and Shekhar. ${ }^{5,7}$ Perhaps, the strongest evidence in favour of the vacuum extractor comes from the results of meta-analyses published by Cochrane Database (1999) which showed that the vacuum device was significantly less likely to cause serious maternal injury than forceps. ${ }^{8}$ There was no significant difference in puerperal complications between forceps and ventouse as seen in Table 3 and Figure 3.
There was no significant difference in the neonatal morbidity in the two groups as seen in Table 4 and Figure 4. Abrasions and bruises were seen in 3 cases of forceps, but these were superficial and disappeared within few hours after birth. There was one neonatal death in the forceps group. However, it was due to respiratory distress in a preterm infant, and cannot be attributed directly to forceps. A study by Prapas showed that the rates of neonatal trauma did not differ significantly between the two groups. ${ }^{9}$ However, most authors agree that serious neonatal injuries are rare with vacuum extraction. ${ }^{10}$

Neonatal well-being assessed by Apgar scores was no different among the groups (Table 5), consistent with other reports. We observed a higher rate of cephalhematoma and jaundice with vacuum extraction in our study, though the difference was not statistically significant

\section{CONCLUSION}

The present study analysed maternal and foetal outcomes in instrumental vaginal deliveries and suggests that ventouse application is associated with significantly less maternal trauma than with forceps. There seems to be no difference in neonatal outcome. The major factor which determines the safety of the instrument is the operator 
rather than the instrument. Either method can be used as long as the operator is skilled with good judgement and expertise. In modern obstetrics, it is seen that the incidence of Forceps delivery is becoming less and less. But obstetrics forceps is a wonderful instrument if used properly after training. A day should not come in Obstetrics where obstetric forceps becomes a museum piece. Therefore, obstetricians should be trained during their residency itself for both types of instrumental vaginal deliveries.

Funding: No funding sources Conflict of interest: None declared

Ethical approval: Not required

\section{REFERENCES}

1. Misra R, Gurunath S. Operative Vaginal Delivery. Ian Donald'S Practical obstetric problems. $7^{\text {th }}$ ed. London: Lloyd-Luke (Medical Books); 2014:550566

2. Unzila A, Ali E. Vacuum-assisted vaginal delivery. Rev Obstet Gynecol. 2015;2(1):5.

3. Cunningham F, Williams J. Operative Vaginal Delivery. Williams obstetrics. $24^{\text {th }}$ ed. New York: McGraw-Hill Professional; 2014:574-586

4. Gei A, Pacheco L. Operative vaginal deliveries: practical aspects. Obstet Gynecol Clini North Am. 2011;38(2):323-349.
5. Singh A, Rathore P. A comparative study of fetomaternal outcome in instrumental vaginal delivery. J Obstet Gynecol India. 2011;61(6):663-6.

6. Vaishnav G, Vaishnav J. Outlet forceps in modern era, A Dangerous instrument or an Art of Obstetrics. Med Sci Int Med J. 2012;1(3):1.

7. Shekhar S, Rana N, Jaswal R. A prospective randomized study comparing maternal and fetal effects of forceps delivery and vacuum extraction. J Obstet Gynecol India. 2012;63(2):116-9.

8. Johnson RB, Menon V. Vacuum extraction versus forceps for assisted vaginal delivery. Cochrane Database Syst Rev. 2000;(2):CD000224.

9. Prapas N, Kalogiannidis I, Masoura S, Diamanti E, Makedos A, Drossou D et al. Operative vaginal delivery in singleton term pregnancies: Short-term maternal and neonatal outcomes. Hippokratia. 2009 Jan;13(1):41-5.

10. Achanna S, Monga D. Outcome of forceps delivery versus vacuum extracton, a review of 200 cases. Singapore Med J. 1994;35:605-8.

Cite this article as: Ahmed MS, Bhalerao AN.

Comparative study of maternal and foetal outcome in instrumental vaginal deliveries. Int J Reprod Contracept Obstet Gynecol 2017;6:4945-9. 